\title{
TIME-LAPSE MONITORING OF SUBSURFACE FLUID FLOW USING PARSIMONIOUS SEISMIC INTERFEROMETRY
}

\author{
Sherif M. Hanafy, KAUST, Thuwal, KSA - Cairo University, Giza, Egypt \\ Jing Li, KAUST, Thuwal, KSA \\ Gerard T. Schuster, KAUST, Thuwal, KSA
}

\begin{abstract}
A typical small-scale seismic survey (such as 240 shot gathers) takes at least 16 working hours to be completed, which is a major obstacle in case of time-lapse monitoring experiments. This is especially true if the subject that needs to be monitored is rapidly changing. In this work, we will discuss how to decrease the recording time from 16 working hours to less than one hour of recording. Here, the virtual data has the same accuracy as the conventional data. We validate the efficacy of parsimonious seismic interferometry with the time-lapse mentoring idea with field examples, where we were able to record 30 different data sets within a 2-hour period. The recorded data are then processed to generate 30 snapshots that shows the spread of water from the ground surface down to a few meters.
\end{abstract}

\section{Introduction}

Refraction tomography is an important imaging tool in earthquake studies (Stein and Wyession, 2003), crustal-mantle imaging (Prodehl and Mooney, 2012), exploration geophysics (Yilmaz, 2001), and engineering seismology (Yilmaz, 2015). Surface waves are often used to estimate the S-wave tomogram for the near surface and engineering applications (Xia et al., 1999; Shapiro et al., 2005; Lin et al., 2008; Socco et al., 2010).

One of the main challenges in the seismic method is the long time to record all the shot gathers. In a 240 shot gather example, where each shot is recorded using 15 stacks for each shot location, the recording time for each shot is about 4 minutes, and to finish recording 240 CSGs we need $(4 * 240=960$ minutes) around 16 hours of shooting time. To decrease the recording time to less than one hour we introduce a method named "Parsimonious Seismic Interferometry" (Hanafy and Schuster, 2016), where densely populated refraction data or surface-wave data can be obtained from two shots on either end of the recording line, and a few infill shot gathers. For refractions, the assumptions are that the first arrivals mainly consist of head waves and direct waves, and a pair of offset shot gathers and several infill shot gathers are recorded over the line of interest. Refraction traveltimes from these shot gathers are picked and spawned into $\mathrm{O}\left(N^{2}\right)$ refraction traveltimes generated by $N$ virtual sources, where $N$ is the number of geophones in the 2D survey. This enormous increase in the number of traveltime picks and associated rays, compared to the many fewer traveltimes from the two opposite and few infill shot gathers, allows for increased model resolution and a better condition number with the system of normal equations. This proposed technique is also valid for surface waves.

Parsimonious seismic surveys save recording time, but at the cost of less slowness resolution and certainty in the estimate of the subsurface model. 


\section{Method}

Assume a source on each end of the recording line and the irregularly layered medium in Figure (1a), where head waves propagate along the interface between the upper and lower layers. There can be lateral velocity variations in the upper medium and there are $N$ evenly spaced geophones on the recording surface between the two sources. The head-wave traveltime from the source at $\boldsymbol{A}$ to a receiver at $\boldsymbol{C}$ is defined as

and the opposite traveltime from $\boldsymbol{D}$ to $\boldsymbol{B}$ is

$$
\tau_{A C}=\tau_{A x^{\prime}}+\tau_{x^{\prime} x}-\tau_{x C}
$$

$$
\tau_{D B}=\tau_{D x}+\tau_{x^{\prime} x}-\tau_{x B}
$$

where $\tau_{x} x^{\prime}$ is the traveltime from $x$ to $x^{\prime}$ along the refraction ray. To create virtual sources and receivers within the array in Figure (1a), we assume that the geophone located at positions $\boldsymbol{C}$ and $\boldsymbol{B}$ are separated at a post-critical distance. Adding equation (1) to equation (2) and subtracting the reciprocal traveltime $\left(\tau_{A D}\right)$ gives the interferometric stationary traveltime from a virtual source (Schuster et al., 2014) located at $\boldsymbol{B}$ to a receiver located at $\boldsymbol{C}$, as shown below

$$
\begin{aligned}
\tau_{C B} & =\tau_{A C}+\tau_{D B}-\tau_{A D}, \\
& =\left[\tau_{A x^{\prime}}+\tau_{x^{\prime} x}-\tau_{x C}\right]+\left[\tau_{D x}+\tau_{x^{\prime} x}-\tau_{x B}\right]-\left[\tau_{A x^{\prime}}+\tau_{x^{\prime} x}-\tau_{x D}\right], \\
& =\tau_{x \prime B}+\tau_{x \prime x}-\tau_{x C} .
\end{aligned}
$$

This equation is also valid for surface waves as shown in Figure 1b. In the surface-wave case, we crosscorrelate the trace $D(\omega)_{C A}$ from a source located at $\boldsymbol{A}$ to a receiver located at $\boldsymbol{B}$ (denoted as solid-red line in Figure 1) with the trace $D(\omega)_{B A}$ (dashed-red line in Figure 1) to cancel the common part. This procedure creates the virtual (solid-blue) trace $D(\omega)_{C B}$ that is corresponding to the surface wave associated with a virtual shot located at $\boldsymbol{B}$ and a receiver located at $\boldsymbol{C}$ :

$$
D(\omega)_{B C}=\sum_{A} D(\omega)_{C A} D(\omega)_{B A}^{*}
$$

a) Parsimonious Seismic Interferometry: Refraction Case

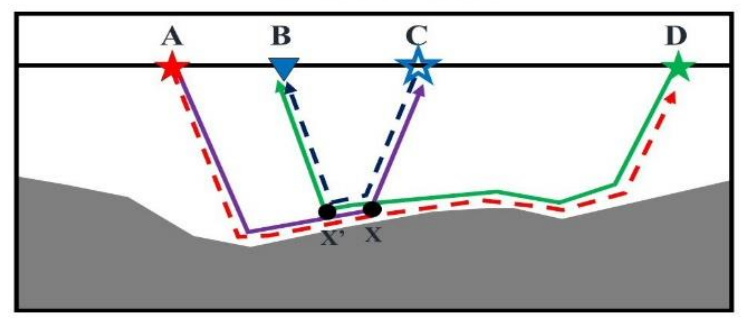

b) Parsimonious Seismic Interferometry: Surface-wave Case

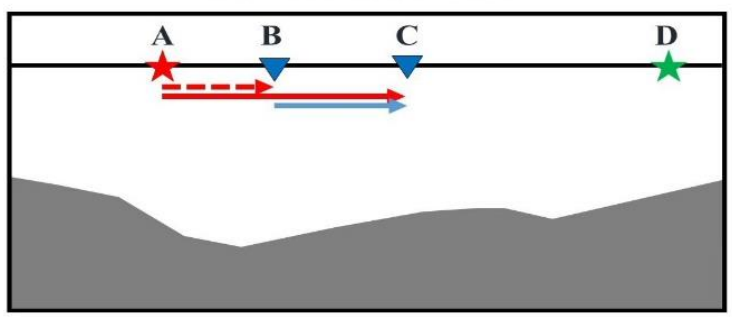

Figure 1: The parsimonious seismic interferometry method explained on a 2-layer model, where the lower layer has faster seismic velocity than the upper layer. a) Shows the refraction case, while b) shows the surface-wave case.

\section{Numerical Results}

The parsimonious seismic interferometry method is tested with synthetic and field data examples. We will show one synthetic example (refraction case) and one field example (time-lapse surface-wave case). For more details see Hanafy and Schuster (2016) and Hanafy and Schuster (2017).

\section{Synthetic Example}

We now test the proposed method on the two-layer model shown in Figure (2a). The first arrival traveltimes are calculated using a finite-difference solution to the 2D eikonal equation (Qin et al., 1992), 
where we created 120 shot gathers with sources located every $5 \mathrm{~m}$ on the surface and 120 receivers share the same positions as the shot points

The traveltimes of the two end-line shot gathers located at $0 \mathrm{~m}$ and $600 \mathrm{~m}$ are inverted to get the P-velocity tomogram in Figure (2b). In this case there is a poor correspondence between the reciprocal tomogram and the actual velocity model. Figure (2c) shows the standard tomogram inverted from all 14,400 calculated traveltimes, which mostly agrees with the actual velocity model.

Equation (3) is then used to compute the virtual traveltimes from the 240 traveltimes associated with the two opposite shot gathers. The result is the creation of $(>14,000)$ virtual traveltimes computed for virtual shots at each of the geophones. These virtual traveltimes are inverted to give the virtual tomogram shown in Figure (2d). This tomogram almost perfectly agrees with the actual model, which is not surprising because there are few diving waves in the model and there is dense illumination in the subsurface by the virtual rays.

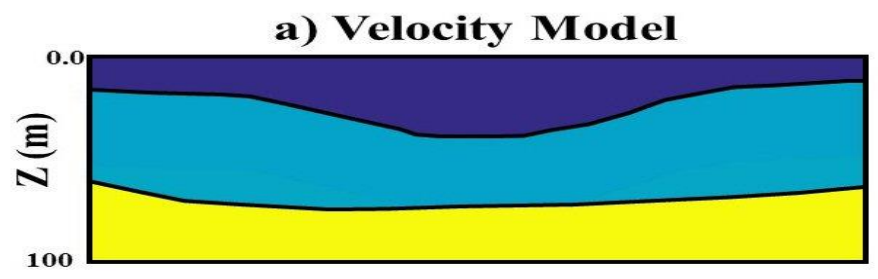

c) Standard Tomogram

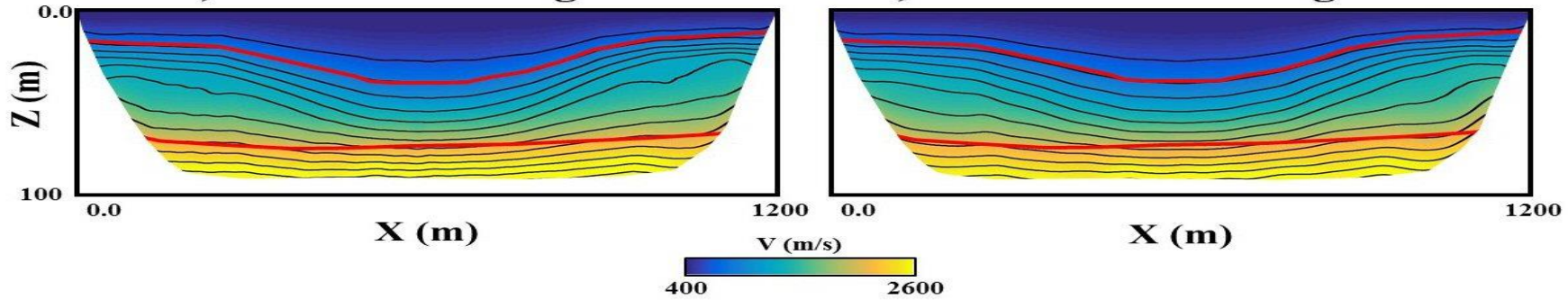

b) Opposite + Infill Tomogram

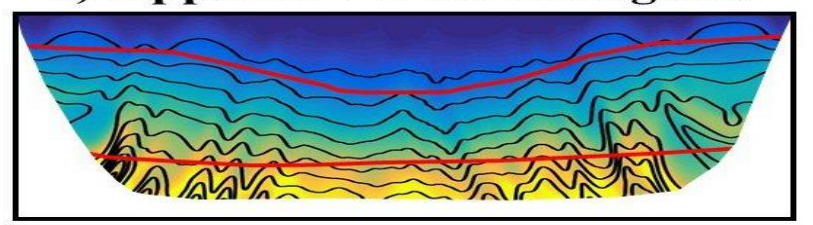

d) Virtual-data Tomogram

Figure 2: a) Two-layer model, b) tomogram inverted from the 240 traveltimes in the two opposite shot gathers, c) standard tomogram inverted from the 14,400 actual traveltimes. d) Virtual tomogram inverted from $(>14 ; 000)$ virtual traveltimes created from the input data consisting of 240 traveltimes.

\section{Time-lapse Field Example}

Seismic time-lapse techniques are limited to large-scale applications, such as hydrocarbon reservoir exploration geophysics (Landro, 2001) or the monitoring of geologic storage reservoirs (Picotti et al., 2012). In the field of near-surface geophysics, the potential application of seismic methods for tracking time-variant processes has been assessed (Jefferson et al., 1998). Recently, the use of seismic surface-wave techniques has been proposed for the detection of varying levels of water table of shallow aquifers, heavy-oil (Pasquet et al., 2015), or the climate effects on earthworks (Bergamo et al., 2016).

The time-lapse approach is now tested using a field data example. A time-lapse water injection experiment is recorded at the KAUST Stadium area, where a line of 72 common shot gathers (CSG) with 72 receivers/CSG and $1 \mathrm{~m}$ shot and receiver intervals are deployed (Figure 3). This data set is recorded as the background data. Then we dumped 500 liters of water at the location of receivers 19 to 23 while data are continuously recorded using the parsimonious interferometry technique. Here, only 5 
shots located at $\mathrm{X}=0,19,37,58$, and 71 meters are recorded. We were able to record 30 different data sets within a 2-hour period.

The virtual CSG for the virtual shot located at $\mathrm{X}=19$ meters is calculated using equation (4) for all 30 time-lapse data sets. Then the dispersion curves of all the data sets are calculated and inverted (Li and Schuster, 2016) to create the phase-velocity profile shown in Figure (4). Figure (4) shows that the 2D phase-velocity profile of the first layer is decreasing with increasing calendar time and increasing water saturation in the near-surface layer.
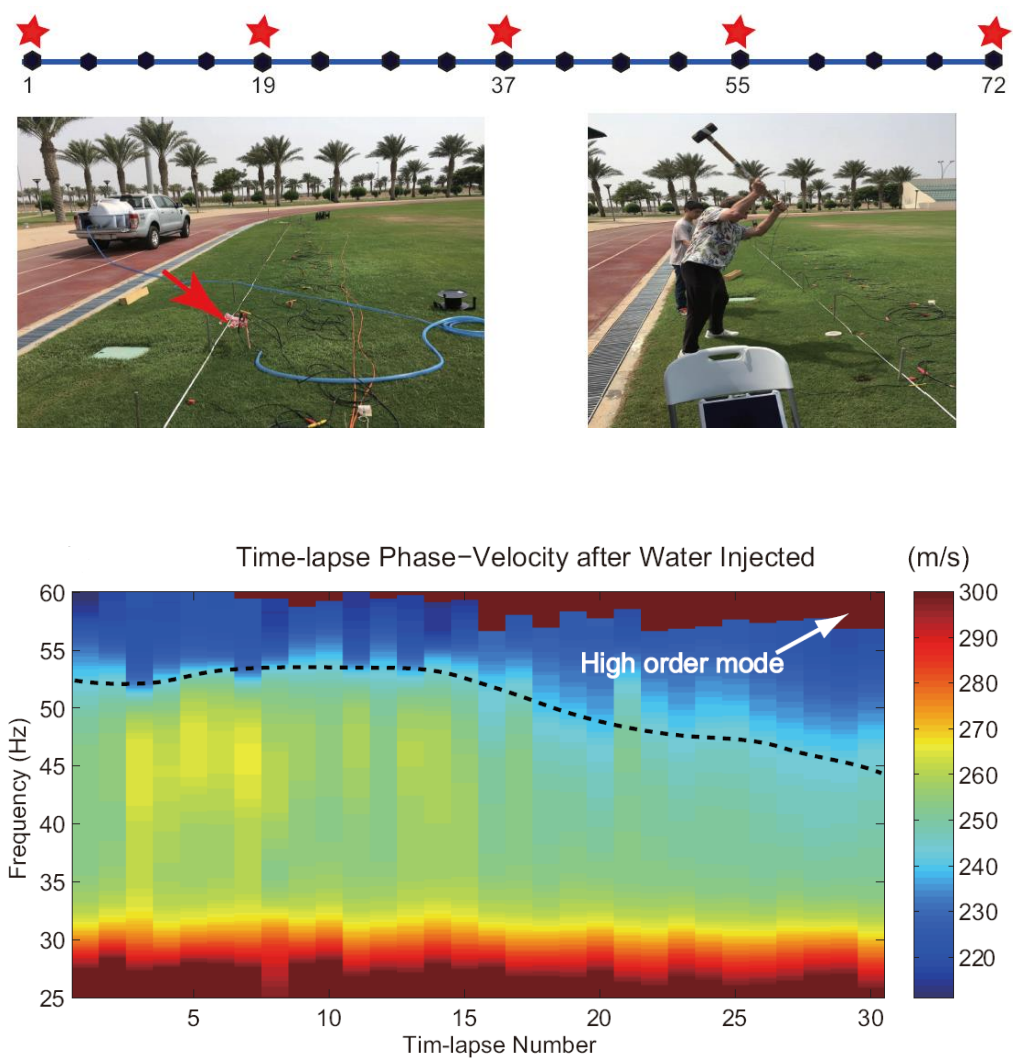

Figure 3: A photo of time-lapse water injection monitoring experiment. Red stars and black circles represents the shot and receiver locations, respectively. The water is injected between $\mathrm{X}$ $=19 \mathrm{~m}$ and $23 \mathrm{~m}$.

Figure 4: Time-lapse surfacewave phase velocity at the water injection location (CSG \# 19). The horizontal axis depicts the time lapse index, where the time difference between two neighboring indices is 4 minutes. The frequency $28 \mathrm{~Hz}$ roughly corresponds to a depth of $5 \mathrm{~m}$ and $56 \mathrm{~Hz}$ roughly corresponds to a depth of $1.5 \mathrm{~m}$.

\section{Conclusions}

The theory of parsimonious seismic interferometry is presented here, where a dense set of virtual refraction traveltimes is computed from refraction traveltimes picked from a pair of opposite shot gathers. In theory, a virtual-shot gather of traveltimes can be computed for a virtual shot at each of the geophones in the 2D reciprocal survey. This means that $\mathrm{O}\left(N^{2}\right)$ shot gathers of virtual traveltimes can be created from virtual shots placed at each of the $N$ geophones.

Tests with synthetic and field data validate that inversion of virtual refraction traveltimes can give tomograms that closely resemble those computed from traveltimes recorded in a dense survey. If the first arrivals are mostly head-wave arrivals, not strong diving waves, then dense 2D refraction surveys might be replaced by inexpensive surveys with as few as two shots placed at each end of the line.

In addition, the time-lapse parsimonious surface-wave monitoring test demonstrates that it is a robust and reliable technique in velocity monitoring over the length of the survey. 


\section{References}

Bergamo, P., B. Dashwood, S. Uhlemann, R. Swift, J. E. Chambers, D. A. Gunn, and S. Donohue, 2016, Time-lapse Monitoring of Climate Effects on Earthworks using Surface Waves, Geophysics, 81, EN1-EN15.

Hanafy, Sherif M. and Gerard T. Schuster, 2016, Parsimonious Refraction Interferometry. SEG Technical Program Expanded Abstracts 2016, 2339-2343.

Hanafy, Sherif M. and Gerard T. Schuster, 2017, Parsimonious Refraction Interferometry, submitted to Geoph. J. Int., under moderate revision.

Jefferson, R. D., D. W. Steeples, R. A. Black, and T. Carr, 1998, Effects of soil-moisture content on shallow-seismic data, Geophysics, 63, 1357-1362

Landro, M., 2001, Discrimination between Pressure and Fluid Saturation Changes from Time Lapse Seismic Data, Geophysics, 66, 836-844.

L, Jing and Gerard T. Schuster, 2016, Skeletonized wave equation of surface wave dispersion inversion, SEG Technical Program Expanded Abstracts 2016, 3630-3635.

Lin, F. C., M. P. Moschetti, and M. H. Ritzwoller, 2008, Surface Wave Tomography of the Western United States from Ambient Seismic Noise: Rayleigh and Love Wave Phase Velocity Maps, Geophysical Journal International, 173, 281-298.

Pasquet, S., L. Bodet, A. Dhemaied, A. Mouhri, Q. Vitale, F. Rejiba, N. Flipo, and R. Guerin, 2015, Detecting Different Water Table Levels in a Shallow Aquifer with Combined P-, Surface and Sh-wave Surveys: Insights from Vp/Vs or Poisson's Ratios, Journal of Applied Geophysics, 113, 38-50.

Picotti, S., J. M. Carcione, D. Gei, G. Rossi, and J. E. Santos, 2012, Seismic Modeling to Monitor CO2 Geological Storage: The Atzbach-Schwanenstadt Gas Field, Journal of Geophysical Research: Solid Earth, 117, DOI, 10.1029/2011JB008540.

Prodehl, C. and W. D. Mooney, 2012, Exploring the Earth's Crust: History and Results of ControlledSource Seismology, Geological Soc. of America, 764 p.

Qin, F., Y. Luo, K. B. Olsen, W. Cai, and G. T. Schuster, 1992, Finite-difference solution of the eikonal equation along expanding wavefronts, Geophysics, 57, 478-487.

Schuster, G. T., Yunsong Huang, Sherif M. Hanafy, Min Zhou, Jianhua Yu, Ola Alhagan, and Wei Dai, 2014, Review on Improved Seismic Imaging with Closure Phase, Geophysics, Vol. 79, No. 5, pp. W11-W25

Shapiro, N. M., M. Campillo, L. Stehly, and M. H. Ritzwoller, 2005, High-resolution Surface-wave Tomography from Ambient Seismic Noise, Science, 307, 1615-1618.

Socco, L. V., S. Foti, and D. Boiero, 2010, Surface-wave Analysis for Building Near-surface Velocity Models Established Approaches and New Perspectives, Geophysics, 75, 75A83-75A102.

Stein, S. and M. Wyession, 2003, An introduction to Seismology, Earthquakes, and Earth Structure, Blackwell Publishing Company, $512 \mathrm{p}$.

Xia, J., R. D. Miller, and C. B. Park, 1999, Estimation of Near-surface Shear-wave Velocity by Inversion of Rayleigh Wave, Geophysics, 64, 691-700.

Yilmaz, O., 2001, Seismic Data Analysis, SEG Publishing, Tulsa, OK, http://dx.doi.org/10.1190/1.9781560801580

Yilmaz, O., 2015, Engineering Seismology: with Applications to Geotechnical Engineering, Society of Exploration Geophysicists, http://dx.doi.org/10.1190/1.9781560803300. 